\title{
Evaluating the effect of inpatient diabetes education on length of stay, readmission rates and mortality rates: a systematic review
}

\author{
ZEESHAN HUSSAIN, ${ }^{1}$ MOHAMMED ALKHARAIJI, ${ }^{1}$ ISKANDAR IDRIS ${ }^{2}$
}

\begin{abstract}
Background: Hospitalised patients with diabetes experience a longer duration of inpatient stay, increased readmission rates and excess mortality compared with patients without diabetes.

Objectives: To determine whether inpatient diabetes education (IDE), provided to hospitalised patients with diabetes, is an effective intervention in improving one or all of the following clinical outcomes: length of stay (LOS), readmission rate and mortality rate.

Methods: A free-text search on MEDLINE, PubMed, CINAHL, BNI and EMBASE was conducted on literature published from the date of each databases' inception to March 2019. In addition, grey literature was used to support the search with the following key terms: 'IDE', 'LOS', 'readmission' and 'mortality', along with their possible substitutes and alternatives combined.

Results: In total, eight studies met the inclusion criteria with a total number of 3,828 participants. Seven studies investigated LOS outcome for which accumulated mean LOS and median LOS were both lower $(16.5 \%$ and $26.67 \%$, respectively) in the IDE group compared with the non-IDE group. Six studies investigated readmittance rates, for which accumulated readmission rate (up to 12 months) was $15.9 \%$ lower in the IDE group than in the non-IDE group. Finally, the mortality rate was $36.6 \%$ lower in the IDE group compared with the non-IDE group, but this was non-significant and only one study reported this outcome.

Conclusion: The findings of this review support the efficacy of an IDE programme in a hospital setting by reducing LOS and readmission rates in patients with diabetes. In addition,
\end{abstract}

Division of Graduate Entry Medicine and Health, University of Nottingham, Nottingham, UK

2 Associate Professor, School of Medicine, University of Nottingham, Nottingham, UK

Address for correspondence: Dr Iskandar Idris Associate Professor, School of Medicine, University of Nottingham, Nottingham, DE22 3DT, UK

E-mail: iskandar.idris@nottingham.ac.uk

https://doi.org/10.15277/bjd.2020.256 a possible trend towards a decreased mortality rate was observed. IDE is therefore recommended to improve clinical outcomes of hospitalised patients with diabetes.

Br J Diabetes 2020;20:96-103

Key words: inpatient diabetes, education, length of stay, readmission rate, mortality, systematic review

\section{Introduction}

The prevalence of diabetes in the UK has increased by about 20\% between 2012 and 2018, ${ }^{1}$ which has translated to an increased number of hospital admissions with patients with diabetes being three times more likely to be hospitalised than similar patients without diabetes. ${ }^{2}$ Admitted patients with diabetes experience a longer length of inpatient stay $(\text { LOS })^{3}$ and have higher rates of readmission ${ }^{4,5}$ and mortality ${ }^{6}$ than patients without diabetes. Collectively, over the past decade this has resulted in an increased utilisation of healthcare resources to manage inpatient diabetes as well as increased occupancy of hospital beds (approximately 1 in 6) by patients with diabetes. ${ }^{7}$ According to Diabetes UK (2019), in 2012 the National Health Service (NHS) had spent in excess of $£ 13$ billion of its healthcare budget on diabetes management and inpatient expenses comprised the majority of this budget ( f9 billion). ${ }^{8}$ Therefore, it is evident that increased LOS and higher rates of readmission and mortality are contributing to the medical expenditures related to inpatient diabetes care.

Inpatient diabetes education (IDE) is considered to be a cornerstone of diabetes care as hospitalisation provides a real opportunity for healthcare staff to address educational deficiencies in patients living with diabetes. The aim of IDE is to reinforce the patients' knowledge and understanding of managing their diabetes outside the secondary care settings. ${ }^{9}$ IDE equips patients with the understanding of the following core elements: correct administration of insulin, including dose and technique of injection; and recognising classic symptoms of dysglycaemia and their appropriate treatment. ${ }^{9}$ IDE is often delivered by a multidisciplinary team which includes diabetologists, diabetes specialist nurses as well as other allied healthcare professionals (diabetes pharmacists, nutritionists and dietitians). However, currently, there is no structured or formal definition of what 
constitutes an IDE, which may present some heterogeneity in IDE contents but also outcomes.

Certain interventions, such as improving diabetes knowledge of the healthcare team ${ }^{10}$ and smooth care transition from hospital to outpatient settings, ${ }^{11}$ have yielded a positive impact on clinical outcomes. However, the overall effectiveness of providing diabetes education to hospitalised patients with diabetes and its impact on clinical outcomes has not been evaluated to date. Therefore, the overarching aim of this systematic review is to ascertain whether IDE improves the following clinical outcomes: (1) inpatient LOS; (2) readmission rate; and (3) mortality rate.

\section{Methods}

\section{Literature search}

The medical literature was electronically searched on the National Institute for Health and Care Excellence (NICE) platform with access to the following five bibliographical databases: CINAHL, MEDLINE, EMBASE, PubMed and BNI. Terms used were related to 'inpatient' and 'diabetes education' in conjunction with 'LOS', 'readmission' and 'mortality' (see Appendix 1 online www.bjd-abcd.com for full search strategy). All types of published articles, with no language restrictions, were searched from the time period between the inception of the databases to March 2019. Furthermore, additional sources such as reference lists of all included studies, Google Scholar and individual journals were hand searched to identify any potential eligible studies that were not detected through the electronic searches.

\section{Selection of studies}

After obtaining the search results, the titles and abstracts of all studies were independently screened to retrieve relevant studies by removing duplicates and irrelevant abstracts. These relevant studies were individually assessed by the author (ZH) and selected to be included in the review if they fulfilled the following criteria: (a) recruited participants in the study had diabetes and were aged 18 years or older; (b) main focus of study is on patient education; (c) intervention takes place in inpatient setting; and (d) results report at least one of the clinical outcomes of interest (ie, LOS, readmission rate or mortality rate). There is currently no formal definition of what constitutes an IDE, and hence there are variations in IDEs for different studies being considered. In summary, the ultimate decision of including or excluding the study was made based on the article title, then the abstract followed by reviewing the full-text article.

\section{Data extraction and synthesis}

Important findings from the eligible studies were independently extracted and subsequently presented in a table format. The use of a table format was preferred since it is well conceived with ease of use and clarity in presenting important findings. ${ }^{12}$

In this review the findings are presented in a table with two main categories: study characteristics and study results. Study characteristics include demographic and descriptive profiles (such as age, gender, sample size, study design and duration of intervention). Study results include the assessed clinical out- comes (ie, LOS, readmission rate and mortality rate). Outcome results, in the form of mean and median for LOS and percentages for readmission and mortality rate, from different studies were extracted (or accumulated using average calculations). Unless otherwise stated, the statistical significance is referred to $p<0.05$ in this review. Due to the marked heterogeneity (mainly clinical) in study results, meta-analysis was not applicable, thus narrative synthesis was selected to discuss the findings. Lastly, Preferred Reporting Items for Systematic Reviews and MetaAnalyses (PRISMA) were followed to structure this review. ${ }^{13}$

\section{Risk of bias assessment}

The methodological quality of all eligible studies was independently assessed by the author $(\mathrm{ZH})$ using the Cochrane Handbook for Systematic Reviews of Intervention tool. ${ }^{14}$ This tool allowed an evaluation of studies for the possibility of the following bias elements: allocation sequence, allocation concealment, blinding (of participants, personnel and outcome assessors), incomplete outcome data and selective outcome for reporting or publication of data. Moreover, the risk of bias figure was created by using Cochrane Review Manager software (Version 5.3, Copenhagen: The Nordic Cochrane Centre, The Cochrane Collaboration, 2014).

\section{Results}

\section{Prisma flow chart summary}

Initially, 1,609 articles (BNI=23, CINAHL=179, EMBASE $=1,015$, MEDLINE=257 and PubMed=135) were identified via an electronic database search. After removing duplicates $(\mathrm{N}=430)$ and irrelevant abstracts ( $N=971), 208$ full-text articles were identified as potentially relevant. These were then subsequently assessed and only four articles met the inclusion criteria. The reasons for excluding 204 articles were as follows: (a) education intervention taking place in outpatient setting; (b) study analysing the data based on patients without the diabetes condition; (c) education not directed towards patients (ie, focus of education is geared towards healthcare staff); and (d) no clinical outcomes of interest were reported. However, grey literature search and reference list checks were also conducted which yielded an additional four study articles. Therefore, a total of eight studies ${ }^{9,15-21}$ became eligible for this review (see PRISMA flow chart in Figure 1).

\section{Study characteristics}

Table 1 shows the study characteristics of the participants $(\mathrm{N}=3,828)$ and summarises the results reported in the eight studies. All studies were published within the last 24 years in six different journals and were carried out in three different countries: USA ( $n=5)$, UK $(n=2)$ and Spain ( $n=1)$. The study design consisted of seven retrospective observational studies and one randomised controlled trial $(\mathrm{RCT})$.

Gender information was reported in four studies, ${ }^{15,16,20,21}$ which showed the percentage of women was lower than men (overall female proportion 46.93\%). Moreover, the mean age from three studies ${ }^{15,19,21}$ was $60.72 \pm 8.7$ years and the median age from four studies ${ }^{16-18,20}$ was 60.43 years, with one study ${ }^{9}$ 
Figure 1. Flow chart of studies included and excluded according to search strategies.
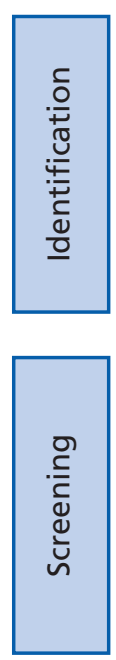

1,609 records identified from database search (BNI, CINAHL, MEDLINE, PubMed and EMBASE)

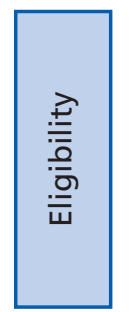

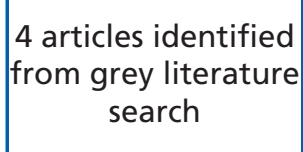

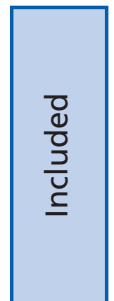

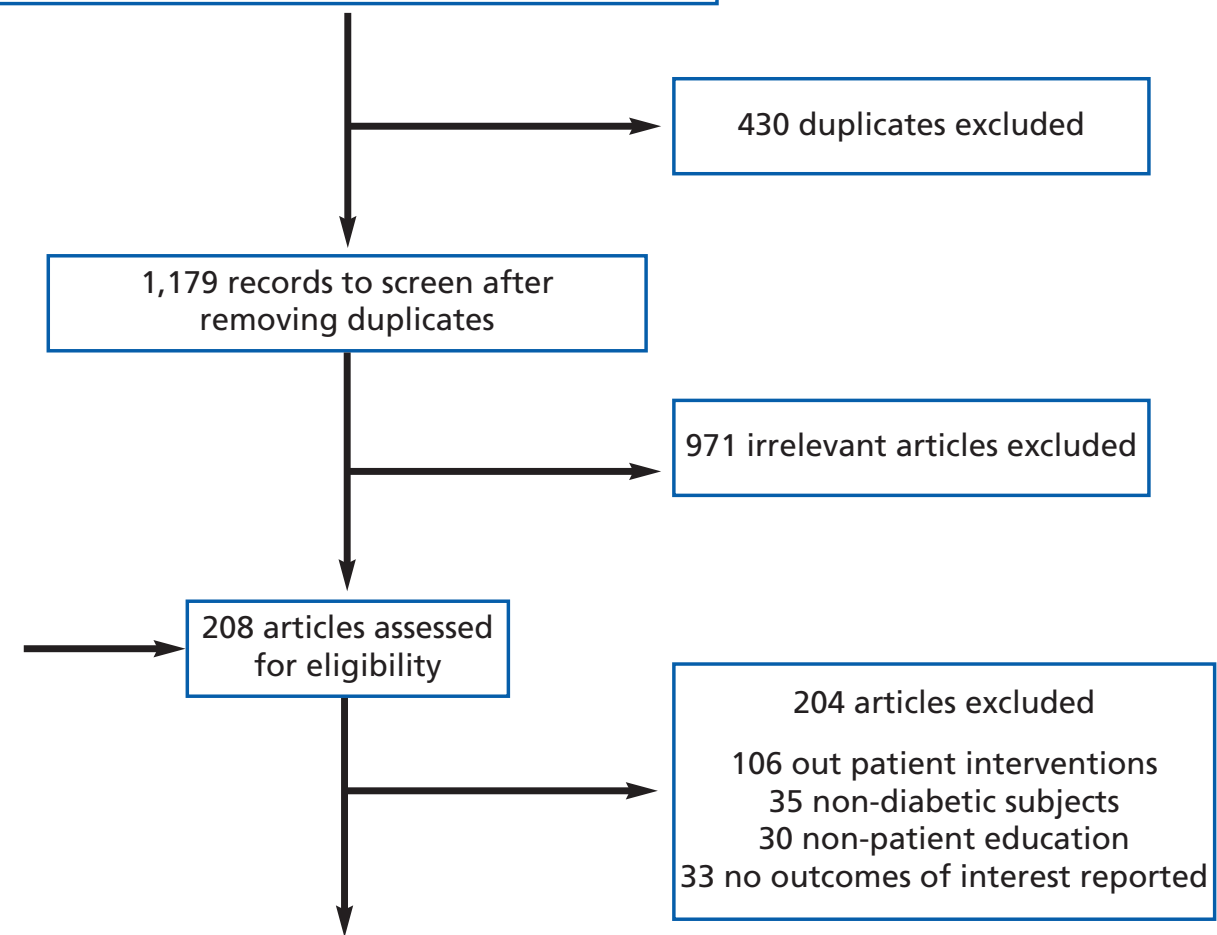

4 articles included from electronic search 4 articles included from grey literature search

Total included study articles, $\mathrm{n}=8$ not documenting the age profile. Sample size ranged from 65 to 2,265 participants, with the duration of the study varying from three to 72 months.

\section{Study results and quality assessment of studies Length of stay}

Of the eight studies included in this review, seven investigated the impact of IDE on LOS. ${ }^{9,15-17,19-21}$ Of these studies, four used mean values $^{15,17,19,21}$ and three used median values $9,16,20$ to present LOS (see Figure 2A and 2B, respectively). An accumulated mean LOS was calculated to be $16.45 \%$ lower in the IDE group compared with the non-IDE group $(5.35 \pm 1.09$ vs $6.40 \pm 2.45$ days, respectively). The calculated greatest mean effect in LOS reduction due to the IDE intervention was $56.1 \%$, which was observed in a study by Levetan et al. ${ }^{19}$ An accumulated median LOS was calculated to be $26.67 \%$ lower in the IDE group compared with the non-IDE group (5.5 vs 7.5 days, respectively). The calculated greatest effect in median LOS reduction due to the IDE intervention was 37.5\%, which was observed in a study by Murphy et al..$^{20}$

\section{Readmission rate}

Of the eight studies included in this review, six investigated the impact of IDE on readmission rates. $9,15,16,18,20,21$ Readmission periods assessed in the included studies varied from 7 days up to 12 months (7 days, 14 days, 30 days, 6 months and 12 months) (see Figure 3). Seven-day readmission was recorded by one study only, ${ }^{15}$ which showed a reduction of almost $60 \%$ $(p<0.01)$ in the readmission rates of the IDE group compared with the non-IDE group (see Figure 3). Fourteen-day readmission was also recorded by one study only, ${ }^{15}$ which demonstrated a non-significant reduction of $38 \%$ in the readmission rates of the IDE group compared with the non-IDE group. Thirty-day readmission was the most popular time period which was observed in five studies. ${ }^{9,15,18,20,21}$ The 30-day readmission rate was over $15 \%$ lower in the IDE group compared with the non-IDE group (accumulated readmittance rate $11.75 \%$ vs $13.85 \%$ ). In addition, two studies ${ }^{18,20}$ showed a statistically significant $(p<0.01)$ reduction in 30-day readmission rates. The 6-month readmission rate was recorded by one study only, ${ }^{18}$ which showed a statistical 
Table 1 Eligible studies presented in chronological order that summarise study characteristics and clinical outcome results

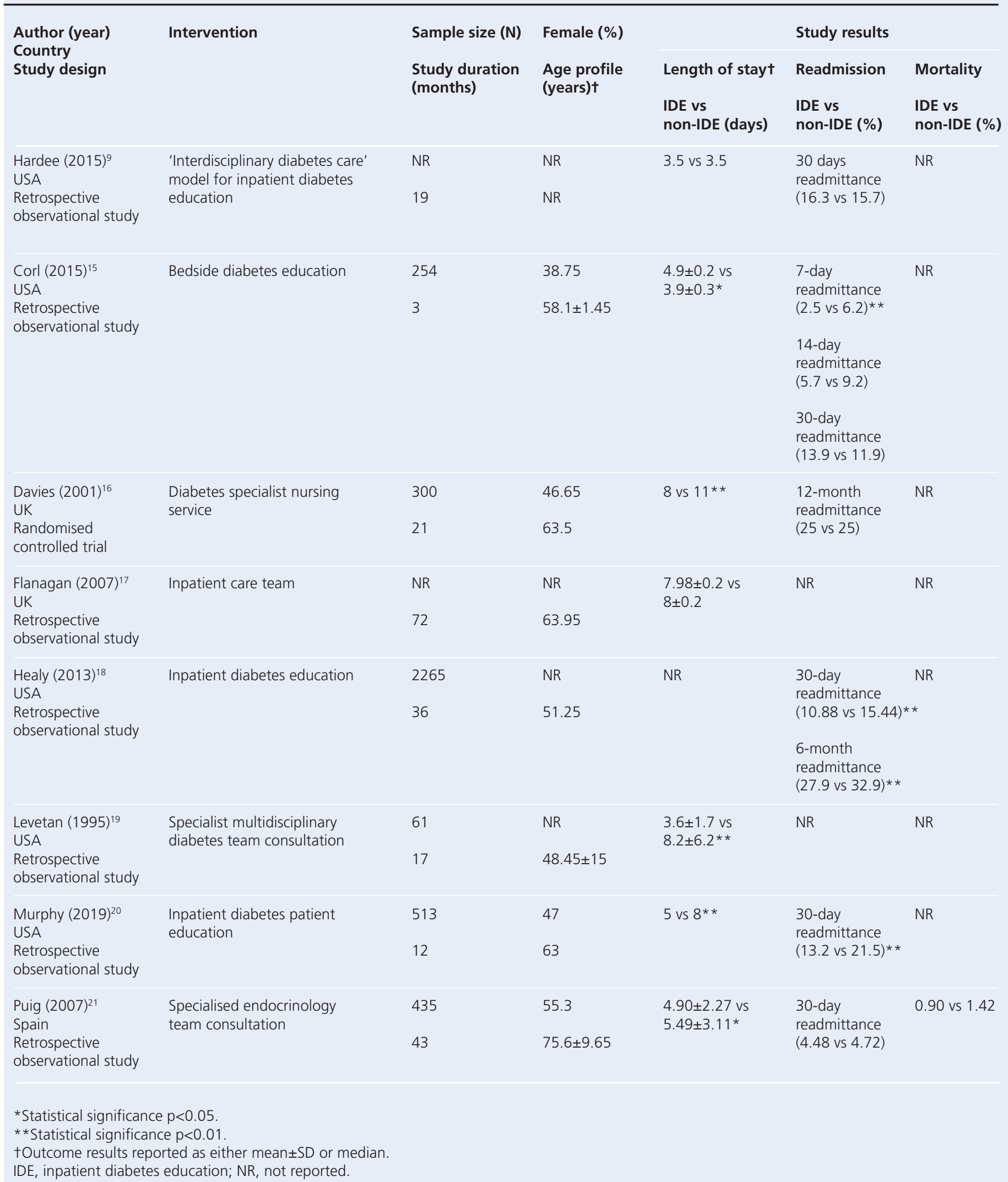


Figure 2. Comparison of the effect of inpatient diabetes education (IDE) versus non-IDE on length of stay in hospital (LOS) among patients with diabetes. (A) LOS is represented as mean values and calculated from four studies. ${ }^{15,17,19,21}$ (B) LOS is represented as median values and calculated from three studies. ${ }^{9,16,20}$

(a) Mean length of inpatient stay in education vs. non-education group

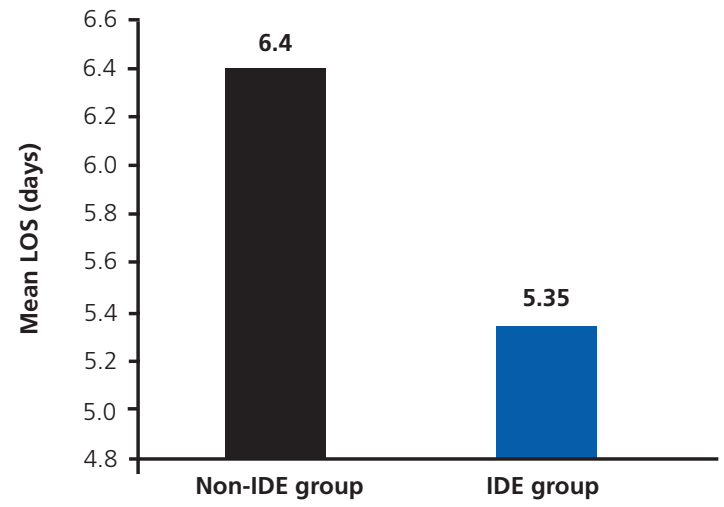

(b) Median length of inpatient stay in education vs. non-education group

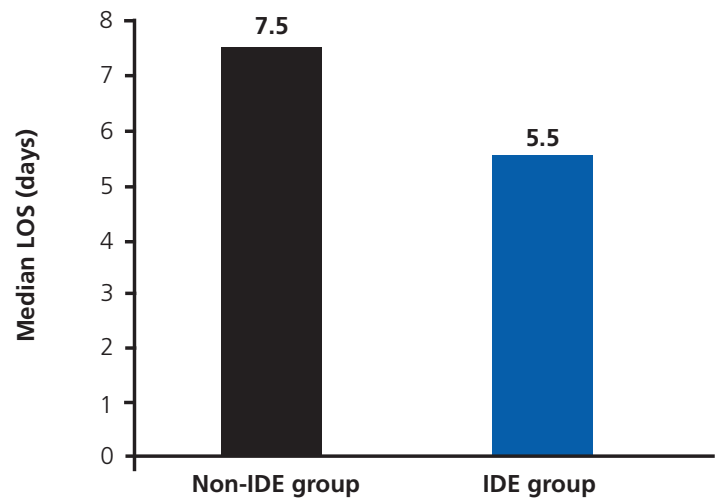

Figure 3. Comparison of the effect of inpatient diabetes education (IDE) versus non-IDE on different periods of readmittance rate among patients with diabetes (up to 12 months). The readmission rate was calculated for the following time periods: 7 days from one study, ${ }^{15} 14$ days from one study, ${ }^{15} 30$ days from five studies, ${ }^{9,15,18,20,21} 6$ months from one study ${ }^{18}$ and 12 months from one study. ${ }^{12}$

Readmittance in education vs non-education group (for periods upto 12 months)

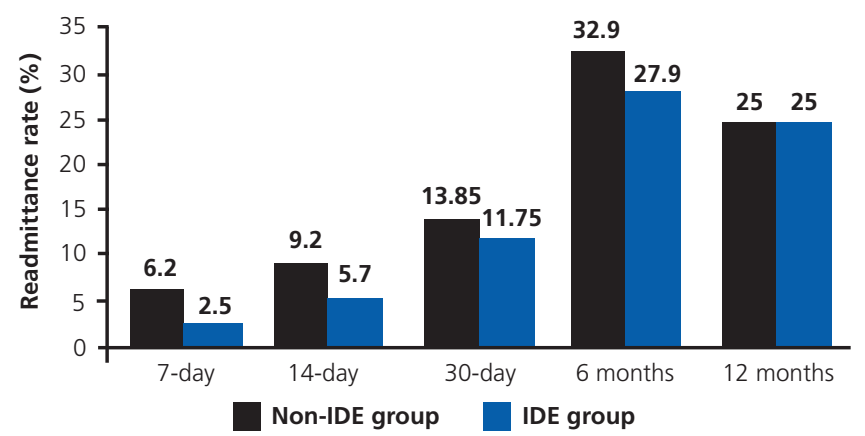

reduction of over $15 \%$ in the readmission rates of the IDE group versus the non-IDE group. Finally, 12-month readmission was recorded by one study only, ${ }^{16}$ where no change in readmission rates was observed ( $25 \%$ reduction across both groups). Overall, for periods up to 12 months, the combined calculated readmission rate was $15.92 \%$ lower in the IDE group than in the nonIDE group (13.32\% vs 15.84\%, respectively) (Figure 4).

\section{Mortality rate}

Of the eight studies included in this review, only one study ${ }^{21}$ investigated the impact of IDE on mortality outcome. In this study, in-hospital mortality was noted to be $36.6 \%$ lower in the IDE group than in the non-IDE group $(0.90 \%$ vs $1.42 \%)$, although
Figure 4. Comparison of the effect of inpatient diabetes education (IDE) versus non-IDE on overall readmittance rate among patients with diabetes. Accumulated readmission rate is calculated from six studies. $9,15,16,18,20,21$

Overall readmittance in education vs non-education group (upto 12 months)

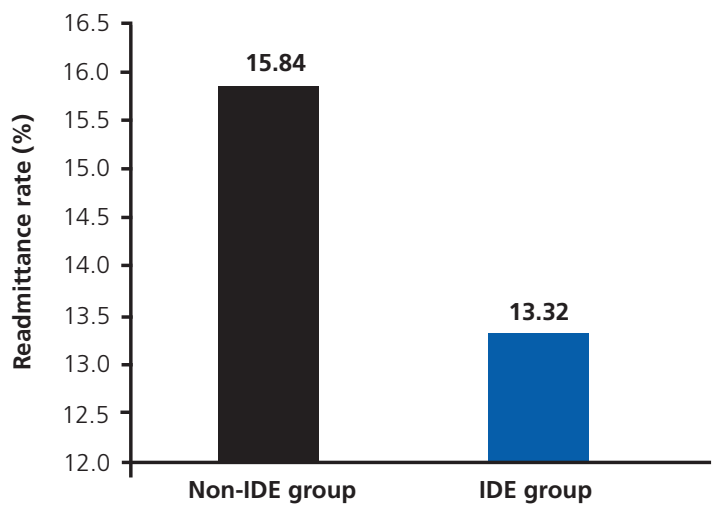

the difference was not statistically significant (95\% confidence interval -5.56 to +4.52 ) (Figure 5).

\section{Quality assessment of studies}

The studies included in the review had different types of bias present (see Appendix 2 online www.bjd-abcd.com). There was a high risk of selection and performance bias in all studies. This was because all the selected studies, except for one RCT, ${ }^{16}$ were designed to retrospectively evaluate the intervention (pre- and post-periods), and therefore randomising participants into separate groups was not feasible. However, the risk of detection bias was low because the outcomes were assessed from historical medical data and therefore the assessor had no influence on 
Figure 5. Comparison of the effect of inpatient diabetes education (IDE) versus non-IDE on mortality rate in patients with diabetes calculated from one study. ${ }^{21}$

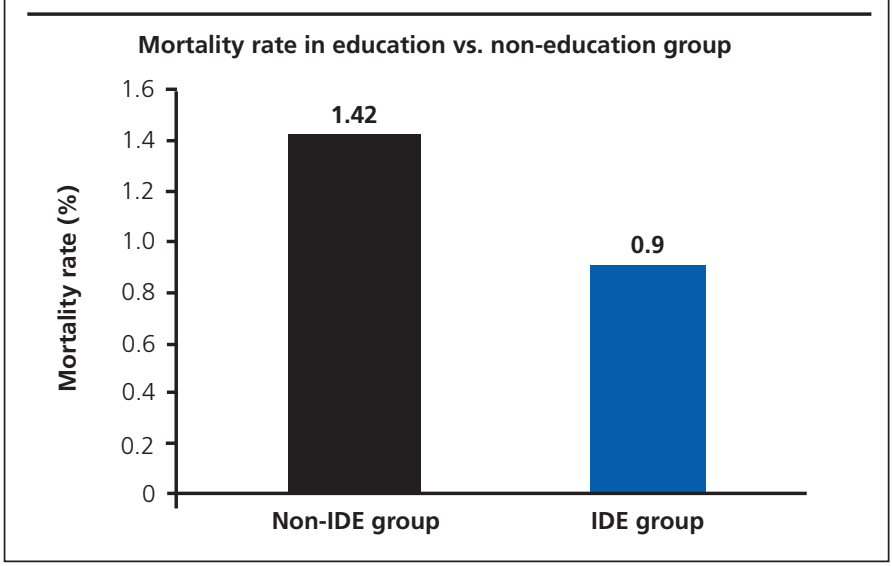

outcomes. Moreover, the attrition and reporting bias was determined to be between low and unclear risk of bias category, which may add to the overall reliability of findings in this review.

\section{Discussion}

The purpose of this review was to determine the impact of IDE on patients' LOS, readmission rate and mortality rate. From the available evidence, the findings indicate that IDE can support reduction in the patients' length of hospital stay and short- to medium-term (7 days up to 6 months) readmission rates. In addition, evidence from only one available study has shown a non-significant reduction in the mortality rate following IDE intervention.

\section{Length of stay}

Providing education to inpatients with diabetes has shown a positive influence on reducing the patients' length of hospital stay. This could be partly explained by the fact that patients receiving the education had improved knowledge and understanding of self-managing their diabetes care. Therefore, this could make the attending physician more confident in expediting the discharge of these patients. Furthermore, IDE would facilitate appropriate post-discharge review in the outpatient. In addition, the successful reduction in LOS could be attributed to an IDE programme being delivered in person (face-to-face contact) by the diabetes staff. Evidence from studies suggests that patients' interaction with educators leads to patients having better understanding of their care needs, treatment option plans and could improve their compliance behaviours. ${ }^{23,24}$

However, a reduction in patients' LOS might not entirely be due to the IDE programme alone. A study by Ahmann ${ }^{25}$ has reported that improvement in inpatient glycaemic control is associated with a reduction in the duration of hospital stay. Moreover, Huang et $a^{26}$ found that the duration from admission to discharge is largely determined by patients' initial severity of illness and intensity of clinical care received. Therefore, it could be likely that patients in the IDE group were acutely ill and suffered fewer co-morbidities and fewer infections than the non-IDE group.

\section{Readmission rate}

Patients who experience shorter LOS have higher rates of early readmissions. ${ }^{27}$ However, our findings in this review are not consistent with this. Our findings indicate that, following IDE, there is a successful reduction in both LOS and short- to medium-term readmission rates (up to 6 months). However, it is evident that the effect of IDE on reducing readmission rates was not sustained at 12 months and suggests that IDE could be supplemented with outpatient educational programmes. ${ }^{28}$ The successful reduction in readmission rates following IDE could be attributed to better self-efficacy skills that patients acquire as part of their education. According to a study by Mohebi et $a^{1},{ }^{29}$ patients with improved self-efficacy skills had better management of their diabetes care at home. Improved self-efficacy is thought to induce motivation and play a vital role in changing the self-care behaviour process, which may lead to improved compliance to diabetes care at home. This could further promote improvement in glycaemic and metabolic control that subsequently delays the progression of long-term complications. ${ }^{30}$ Moreover, the input from IDE may improve patients' understanding of dysglycaemia, which could encourage patients to improve adherence to diabetes nutrition guidelines as well as their antidiabetic medications (ie, correct administration of insulin and sulfonylureas). ${ }^{31,32}$ Lastly, the calculated average age of patients in our review was over 60 years across both groups. Therefore, it is important to highlight that rehospitalisation among these individuals could be due to nondiabetes-related co-morbidities as there is growing evidence of an increasing prevalence of co-morbidities among people aged $>55$ years. ${ }^{33}$ Older adults are more susceptible to the adverse effects of elevated blood glucose levels due to hyperglycaemicinduced immune defects and the use of corticosteroids coupled with age-associated senescence. ${ }^{34}$

\section{Mortality rate}

In this review only one study was eligible for investigating the impact of IDE on mortality, which showed that inpatients with IDE had a lower mortality rate than those who did not receive IDE $(0.9 \%$ vs $1.42 \%)$. The literature on the effect of diabetes education on mortality is scarce, but the available information indicates that the predictor of high mortality is attributed to poor inpatient glycaemic and metabolic control. ${ }^{35}$ Furthermore, our study findings on mortality rate are in agreement with those of McHugh et al, ${ }^{36}$ who observed a significantly lower mortality rate in patients with good glycaemic control compared with poor glycaemic control ( $9 \%$ vs $16 \%, p=0.01$ ). Moreover, frequent hyperglycaemic episodes in hospital have been shown to be associated with increased complications, morbidity and in-hospital mortality. ${ }^{37}$ Therefore, it is important to control glycaemic and metabolic levels of inpatients with diabetes to achieve improved outcomes in mortality rate.

\section{Limitations and conclusion}

The strength of this review lies in broad inclusion and limited exclusion criteria applied to capture studies that represented our well-defined research question. To minimise the potential of 


\section{Key messages}

- Although, a reduction in LOS and rates of readmission and mortality were observed following IDE, it is difficult to establish how much education or timing of education per se corresponds to the reduction in these clinical outcomes

- The heterogeneity of outcomes in different studies may be due to differences in education and educators

- Following IDE, there was a reduction in LOS and short- to medium-term (up to 6 months) readmission rates. Only one study reported on the effect of IDE on mortality rate, which showed a reduction

missing any relevant articles, we conducted an additional search from sources other than the databases on the NICE platform. Despite this, only a few relevant studies were identified, which highlights the dearth of evidence that surrounds this important clinical issue. It is important to interpret this as a lack of evidence rather than evidence of no effect.

Although, a reduction in LOS and rates of readmission and mortality were observed following IDE, it is difficult to establish how much education per se corresponds to the reduction in these clinical outcomes. It is also unclear whether early introduction of IDE has a beneficial impact on these clinical outcomes because the stage at which education was delivered to inpatients has not been recorded in the included studies. To clarify this, robustly designed studies that record pre- and post-intervention knowledge of patients who receive IDE is required. This will identify any improvement in diabetes knowledge of patients from their admission to discharge. Furthermore, studies could also investigate factors responsible for the selection of patients and the level of patient satisfaction from IDE. For example, studies may be associated with a selection bias - that is, patients who are recruited may be less unwell, have better cognitive function and perhaps fewer co-morbidities - which may have an impact on their LOS and mortality risk. Furthermore, studies have not adjusted for patient age, diabetes duration or types of diabetes. Also, the cause of admission was not stated in all studies, which may have an impact on the likelihood of readmission if the problem is a recurrent problem (eg, gastroparesis and diabetic ketoacidosis, infective foot ulcers, etc). The heterogeneity of outcomes in different studies may be due to differences in education and educators. This raises the importance of developing a standard structured education for inpatients with diabetes. However, this may be difficult due to differences in patients' needs and knowledge gap during their hospital stay.

All studies in the review, apart from the $\mathrm{RCT}^{16}$ consisted of a before-and-after design which is predisposed to time-related changes. ${ }^{38}$ Some studies ${ }^{39,40}$ have shown that hospitalised patients with diabetes still remain uneducated about their con- dition at discharge because certain barriers prevent the delivery of IDE (such as acute illness, cost of delivering education and a decrease in the number of staff with specialised knowledge of diabetes). ${ }^{41}$ Patients with acute illnesses have limited exposure to educators in hospitals, so these patients are less likely to have adequate time to receive comprehensive education prior to discharge. ${ }^{42}$

Despite literature being scarce in the area of inpatient education, the reviewed evidence for the IDE suggests a positive effect on clinical outcomes. Following IDE, there was a reduction in LOS and short- to medium-term (up to 6 months) readmission rates. Only one study reported on the effect of IDE on mortality rate, which showed a reduction. To explore these clinical outcomes further, patient satisfaction as well as pre- and postassessment scores of diabetes knowledge is encouraged, which will profoundly contribute to the current literature of IDE.

\section{Conflict of interest None. Funding None.}

\section{References}

1. Diabetes UK (2018). Diabetes prevalence 2018. Available at: https://www.diabetes.org.uk/professionals/position-statementsreports/statistics/diabetes-prevalence-2018 (accessed April 2019).

2. Jiang HJ, Stryer D, Friedman B, Andrews R. Multiple hospitalizations for patients with diabetes. Diabetes Care 2003; 26(5):1421-6. https://doi.org/10.2337/diacare.26.5.1421

3. Comino EJ, Harris MF, Islam MD, et al. Impact of diabetes on hospital admission and length of stay among a general population aged 45 years or more: a record linkage study. BMC Health Services Res 2015;15(1):1-13. https://doi.org/10.1186/s12913-014-0666-2

4. Ostling S, Wyckoff J, Ciarkowski SL, et al. The relationship between diabetes mellitus and 30-day readmission rates. Clin Diabetes Endocrino/ 2017;3(3). https://doi.org/10.1186/s40842-016-0040-x

5. Zapatero A, Gomez HR, Gonzalez N, et al. Frequency of hypoglycemia and its impact on length of stay, mortality, and short-term readmission in patients with diabetes hospitalized in internal medicine wards. Endocrine Pract 2014;20(9):870-5. https://doi.org/10.4158/EP14006.OR

6. Umpierrez GE, Isaacs SD, Bazargan N, et al. Hyperglycemia: an independent marker of in-hospital mortality in patients with undiagnosed diabetes. J Clin Endocrinol Metab 2002;87(3):978-82. https://doi.org/10.1210/ jcem.87.3.8341

7. Diabetes UK (2017). Inpatient care for people with diabetes. Available at: https://www. diabetes.org.uk/professionals/position-statements-reports/specialist-care-for-children-and-adults-and-complications/inpatient-care-forpeople-with-diabetes (accessed April 2019).

8. Diabetes UK (2019). Cost of diabetes. Available at: https:// www.diabetes.co.uk/cost-of-diabetes.html (accessed April 2019).

9. Hardee SG, Osborne KC, Njuguna N, et al. Interdisciplinary diabetes care: a new model for inpatient diabetes education. Diabetes Spectr 2015; 28(4):276-82. https://doi.org/10.2337/diaspect.28.4.276

10. Young JL. Educating staff nurses on diabetes: knowledge enhancement. Medsurg Nurs 2011;20(3):143-6.

11. Cook C, Seifert KM, Hull BP, et al. Inpatient to outpatient transfer of diabetes care: planning for an effective hospital discharge. Endocr Pract 2009;15(3):263-9. https://doi.org/10.4158/EP.15.3.263

12. Conway A, Clarke MJ, Treweek S, et al. Summary of findings tables for communicating key findings of systematic reviews. Cochrane Database of Systematic Rev 2017;2:MR000044. https://doi.org/10.1002/ 14651858.MR000044

13. Moher D, Liberati A, Tetzlaff J, Altman DG, PRISMA Group. Preferred Reporting Items for Systematic Reviews and Meta-Analyses: the PRISMA statement. Ann Intern Med 2009;151(4):264-9. https://doi.org/10.7326/ 0003-4819-151-4-200908180-00135 
14. Higgins J, Green S. Cochrane Handbook for Systematic Reviews of Interventions [Version 5.1.0]. The Cochrane Collaboration. 2011. Available at: http://handbook-5-1.cochrane.org (accessed April 2019).

15. Corl DE, Guntrum PL, Graf L, et al. Inpatient diabetes education performed by staff nurses decreases readmission rates. AADE in Practice 2015;3(2):18-23.

16. Davies M, Dixon S, Currie CJ, Davis RE, Peters JR. Evaluation of a hospital diabetes specialist nursing service: a randomized controlled trial. Diabet Med 2001;18(4):301-7. https://doi.org/10.1046/j.1464-5491.2001.00470.x

17. Flanagan $D$, Moore $E$, Baker S, Wright $D$, Lynch P. Diabetes care in hospital - the impact of a dedicated inpatient care team. Diabet Med 2008; 25(2):147-51. https://doi.org/10.1111/j.1464-5491.2007.02326.x

18. Healy SJ, Black D, Harris C, Lorenz A, Dungan KM. Inpatient diabetes education is associated with less frequent hospital readmission among patients with poor glycemic control. Diabetes Care 2013;36(10):2960-7. https://doi.org/10.2337/dc13-0108

19. Levetan CS, Salas JR, Wilets IF, Zumoff B. Impact of endocrine and diabetes team consultation on hospital length of stay for patients with diabetes. Am J Med 1995;99(1):22-8. https://doi.org/10.1016/s0002-9343(99)80100-4

20. Murphy JA, Schroeder MN, Ridner AT, et al. Impact of a pharmacy-initiated inpatient diabetes patient education program on 30-day readmission rates. J Pharmacy Pract 2019 Feb 2017;897190019833217. https://doi.org/ https://doi.org/10.1177/0897190019833217

21. Puig J, Supervia A, Marquez MA, et al. Diabetes team consultation: Impact on length of stay of diabetic patients admitted to a short-stay unit. Diabetes Res Clin Pract 2007;78(2):211-6. https://doi.org/0.1016/ j.diabres.2007.03.010

22. Burke SD, Sherr D, Lipman RD. Partnering with diabetes educators to improve patient outcomes. Diabetes Metab Syndr Obes 2014;7:45-53. https://doi.org/10.2147/DMSO.S40036

23. Heisler M, Bouknight RR, Hayward RA, Smith DM, Kerr EA. The relative importance of physician communication, participatory decision making, and patient understanding in diabetes self-management. J Gen Intern Med 2002;17(3):243-52. https://doi.org/10.1046/j.1525-1497.2002.10905.x

24. Quinn CC, Royak SR, Lender D, et al. Patient understanding of diabetes selfmanagement: participatory decision-making in diabetes care. J Diabetes Sci Techno/ 2011;5(3):723-30. https://doi.org/10.1177/ 193229681100500327

25. Ahmann A. Reduction of hospital costs and length of stay by good control of blood glucose levels. Endocr Pract 2004;10(2):53-6. https://doi.org/ 10.4158/EP.10.S2.53

26. Huang D, Xie L, Qiu Y. Analysis of factors affecting the length of hospital stay for patients with diabetes. Exp Clin Endocrinol Diabetes 2015; 124(1):5-10. https://doi.org/10.1055/s-0035-1565059

27. Ashton CM, Del Junco DJ, Souchek J, Wray NP, Mansyur CL. The association between the quality of inpatient care and early readmission: a meta-analysis of the evidence. Med Care 1997;35(10):1044-59. https://doi.org/ 10.1097/00005650-199710000-00006

28. Duncan I, Ahmed T, Li QE, et al. Assessing the value of the diabetes educator. Diabetes Educ 2011;37(5):638-57. https://doi.org/10.1177/ 0145721711416256

29. Mohebi S, Azadbakht L, Feizi A, Sharifirad G, Kargar M. Review the key role of self-efficacy in diabetes care. J Educ Health Promot 2013;2:36. https://doi.org/10.4103/2277-9531.115827

30. King P, Peacock I, Donnelly R. The UK Prospective Diabetes Study (UKPDS): clinical and therapeutic implications for type 2 diabetes. Br J Clin Pharmacol 1999;48(5):643-8. https://doi.org/10.1046/j.1365-2125.1999.00092.x

31. Arifulla M, John $\sqcup$, Sreedharan J, et al. Patients' adherence to anti-diabetic medications in a hospital at Ajman, UAE. Malaysian J Med Sci 2014; 21(1):44-9.

32. Aminde LN, Tidong M, Ngwasiri CA, et al. Adherence to antidiabetic medication and factors associated with non-adherence among patients with type-2 diabetes mellitus in two regional hospitals in Cameroon. BMC Endocr Disord 2019;19(1):35. https://doi.org/10.1186/s12902-019-0360-9

33. Hajat $C$, Stein $E$. The global burden of mulriple chronic conditions: a narrative review. Prev Med Rep 2018;12:284-93. https://doi.org/10.1016/ j.pmedr.2018.10.008

34. Szerszen A, Seminara DP, Castellanos MR. Glucose control in the hospitalised elderly - a concern not just for patients with diabetes. Geriatrics 2009;64(6):18-20.

35. Gerard SO, Ritchie J. Challenges of inpatient glycemic control. J Nurs Care Qual 2017;32(3):267-71. https://doi.org/10.1097/NCQ.0000000000000257

36. McHugh MD, Shang J, Sloane DM, Aiken LH. Risk factors for hospital-acquired "poor glycemic control " : a case-control study. Int J Qual Health Care 2011;23(1):44-51. https://doi.org/10.1093/intqhc/mzq067

37. Newton CA, Young S. Financial implications of glycemic control: results of an inpatient diabetes management program. Endocr Pract 2006;12(3):438. https://doi.org/10.4158/EP.12.S3.43

38. Daly S, Campbell DA, Cameron PA. Short-stay units and observation medicine: a systematic review. Med J Aust 2003;178(11):559-63.

39. Levetan CS, Passaro M, Jablonski K, Kass M, Ratner RE. Unrecognized diabetes among hospitalized patients. Diabetes Care 1998;21(2):246-9. https://doi.org/10.2337/diacare.21.2.246

40. Roman SH, Chassin MR. Windows of Opportunity to improve diabetes care when patients with diabetes are hospitalised for other conditions. Diabetes Care 2001;24(8):1371-6. https://doi.org/10.2337/diacare.24.8.1371

41. Beagley L. Education patients: understanding barriers, learning styles, and teaching techniques. J Perianesth Nurs 2011;26(5):331-7. https://doi.org/ 10.1016/j.jopan.2011.06.002

42. Nettles AT. Patient education in the hospital. Diabetes Spectr 2005;18(1): 4-48. https://doi.org/10.2337/diaspect.18.1.44

\title{
Gestational diabetes: screening uptake, current challenges and the future - a focused review
}

\author{
ELPIDA VOUNZOULAKI, KAMLESH KHUNTI, BEE K TAN, MELANIE J DAVIES, CLARE L GILLIES
}

(Br J Diabetes 2020;20:9-14 https://doi.org/10.15277/bjd.2020.236)

In the article listed above, Table 2 - Studies reporting post-partum screening rates and determinants in the UK (page 11), there is a footnote indicating a study that was from Ireland. This study was by Carmody et al and not McGovern et al, which appeared in the printed issue. The correct version of this can be found online www.bjd-abcd.com/index.php/bjd/article/view/505/743 
Appendix 1. Search strategy performed on National Institute for Health and Care Excellence (NICE) platform.

((((educat* OR "diabetes educat*" OR "diabetes under*" OR "diabetes schoo/*"
OR "diabetes knowledge" OR "diabetes tutor*") AND ("hospitali* patient*" OR
hospitali* OR inpatient* OR "secondary care" OR admit* OR ward)) AND
("clinical outcomes" OR LOS OR "length of stay" OR "duration of stay" OR
readmission OR readmit* OR rehospitali* OR mortality OR death)) AND (diabetes
OR TIDM OR T2DM)).ti,ab

Appendix 2. Risk of bias graph: author $(\mathrm{ZH})$ judgements about each risk of bias item presented as percentages across all included studies.

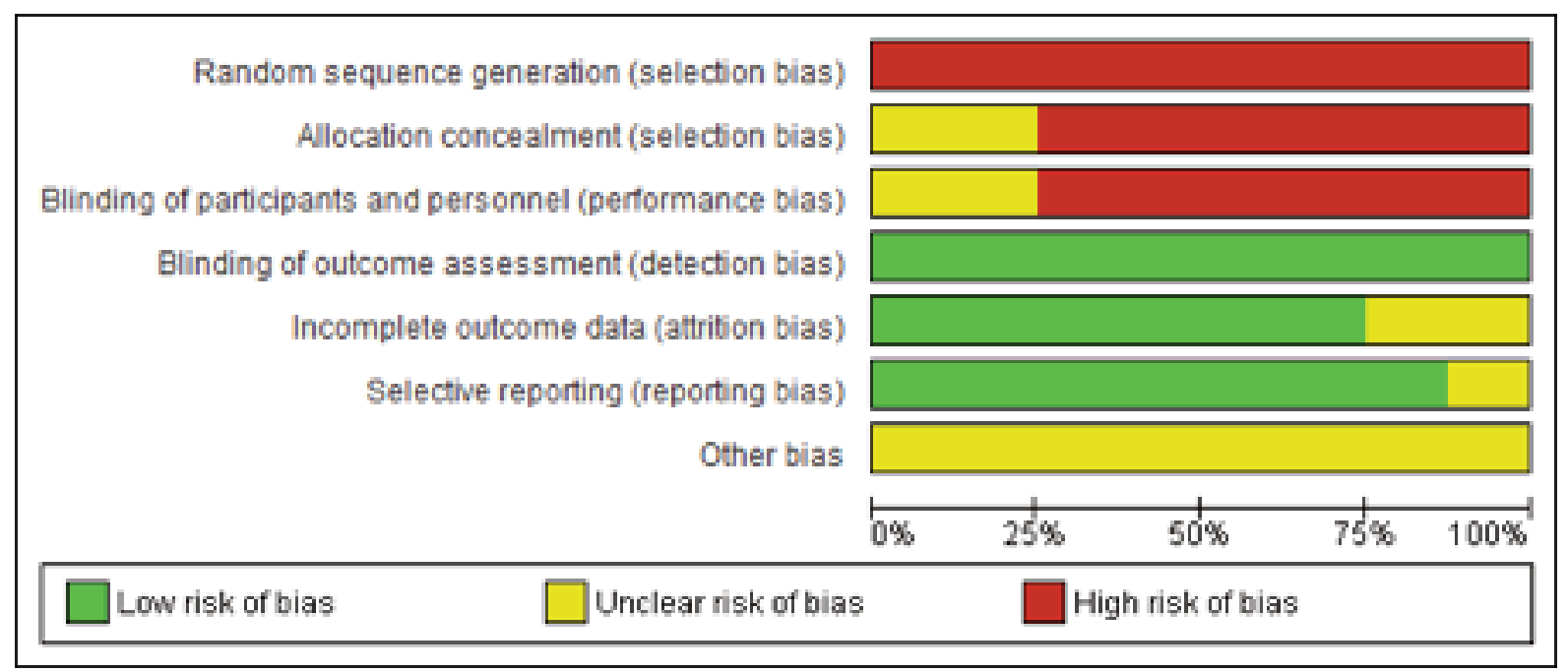

